\title{
Electromagnetic Scattering from Short Branching Vegetation
}

\author{
Tsenchieh Chiu and Kamal Sarabandi, Fellow, IEEE
}

\begin{abstract}
A polarimetric coherent electromagnetic scattering model for short branching vegetation is developed in this paper. With the realistic structures that reasonably describe the relative positions of the particles, this model is able to consider the coherent effect due to the phase difference between the scattered fields from different particles, and account for the second-order, near-field interaction between particles, to which the relative positions and orientation of the particles are essential. The model validation with measurements is also presented, and excellent agreement is obtained. The polarimetric radar backscatter measurements for soybean plants using truck-mounted scatterometers were conducted at L-band and C-band under different soil-moisture conditions. Through an extensive ground truth, the important plant and rough surface parameters such as the soil moisture and surface roughness, vegetation dielectric constant, and geometry of the soybean plants, were characterized for model verification. It is found that the second-order near-field scattering is significant at C-band for fully grown soybeans due to the high vegetation particle density, and at $L$-band, the contribution from the second-order near field is negligible. The coherence effect is shown to be important at $\mathrm{L}$-band and to a much lesser extent at $\mathrm{C}$-band. This model is then used to demonstrate its ability for estimating the physical parameters of a soybean field, including soil moisture from a polarimetric set of AIRSAR images.
\end{abstract}

\section{INTRODUCTION}

M ICROWAVE remote sensing has evolved into an important tool for monitoring the atmosphere and surface of the earth. Electromagnetic waves at microwave frequencies are able to penetrate more deeply into vegetation and therefore, retrieving parameters of vegetation and underlying ground surfaces has become one of the major applications of microwave remote sensing. With the advent of polarimetric synthetic aperture radars (SAR's) and the development of radar polarimetric techniques, microwave remote sensing has attained significant prominence. While a large amount of data can be collected very efficiently, there are still difficulties in accurately predicting the physical parameters of the targets from the collected radar information. To accomplish this task, a necessary step is to construct a high-fidelity scattering model by which the relationship between all targets' physical parameters to the radar backscatter can be established.

In the early vegetation scattering models, the vegetation medium was simplified in terms of a homogeneous random

Manuscript received January 14, 1998; revised February 5, 1999. This work was supported by NASA Contract NAGS-4939 and Jet Propulsion Laboratory Contract JPL-958749.

The authors are with the Department of Electrical Engineering and Computer Science, University of Michigan, Ann Arbor, MI 48109-2122 (e-mail: tcchiu@eecs.umich.edu).

Publisher Item Identifier S 0196-2892(00)02480-3. medium, and the single scattering theory was applied to account for the scattering and propagation in the random medium [1]-[3]. For example, in [1], a forest stand is represented in terms of a two-layer random medium, including a crown layer composed of randomly oriented cylinders and disks representing branches and leaves and a trunk layer containing nearly vertical cylinders representing tree trunks below the crown layer. Although these models are capable of predicting the scattering behavior of vegetation qualitatively, they are incapable of predicting the scattering behavior quantitatively due to their simplifying assumptions. An important feature of a high fidelity scattering model is to preserve the structure of vegetation as different species of vegetation have their own unique structures, which are expected to exhibit their own scattering behaviors. An important effect of the vegetation structure is the coherence effect caused by the relative position of the vegetation particles that produce certain interference patterns. It is shown that the coherence effects caused by the vegetation structure become more significant at lower frequencies [4]. In the remote sensing of vegetation-covered terrain, where the underlying soil surface is the target of interest, low microwave frequencies are recommended and therefore, the coherence effects must be carefully accounted for. The model developed by Yueh et al. [5] may be among the first to address the coherence effects caused by the vegetation structure. In their scattering model for soybeans, a two-scale branching vegetation structure was constructed, and the scattered fields from particles were added coherently. Lin et al. [6] also proposed a coherent scattering model for forest canopies in which rather realistic tree-like structures are constructed using the fractal theory. In both models, the scattering solutions are formulated using the single scattering theory.

Another important issue in modeling the scattering from vegetation is the effect of the multiple scattering among vegetation particles. Vegetation particles are usually arranged in clusters within a single plant such as leaves around end branches and branches around main stems and trunks. Therefore, a vegetation medium may be appropriately considered as locally dense. In such cases, the near-field multiple scattering is strong and may significantly affect the overall response. To accurately evaluate the near-field interaction, the realistic description of the relative positions and orientations of the vegetation particles and accurate and efficient scattering formulations are required. In recent years, some advanced scattering solutions that account for the near-field interaction between scatterers have been presented [7], [8]. However, vegetation scattering models that can handle the near-field interaction with realistic vegetation structures have not been developed yet. The evaluation of the near- 


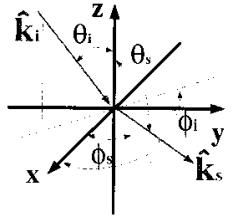

Fig. 1. Definition of the incident and scattering angles.

field interaction is usually numerically intensive, considering the huge number of particles in the medium.

In this paper, a scattering model for soybeans is presented that incorporates realistic computer-generated vegetation structures and accounts for the second-order near-field scattering interaction. Soybeans are erect branching plants composed of components that can often be found in many vegetation stems, branches, leaves, and fruits (pods), arranged in a very well-defined manner. Hence, it is very appropriate for studying the effect of the vegetation structure on the radar backscatter. Also, because of its moderate number of particles, the computation of the second-order near-field interaction is not formidable. In addition, from the experimental point of view, the dimensions of soybean plants are small enough to allow for conducting controlled experiments using truck-mounted scatterometers. Due to the uniformity of the plants and underlying soil surface, gathering the ground truth data is rather simple. The paper is organized as follows: Section II gives the theoretical description of the model, including the vegetation structure modeling and the scattering solution. In Section III, the experimental procedures using the University of Michigan truck-mounted scatterometer and AIRSAR are discussed. Finally, in Section IV, model validation using the measured data and a sensitivity analysis are presented.

\section{THEORETICAL ANALYSIS}

Consider a global coordinate system with $x-y$ plane parallel to a horizontal ground plane and $z$-axis along the vertical direction, as shown in Fig. 1. Suppose a plane wave given by

$$
\boldsymbol{E}^{\boldsymbol{i}}(\boldsymbol{r})=\boldsymbol{E}_{\mathbf{0}}^{\boldsymbol{i}} e^{i k_{0} \hat{k}_{i} \cdot \boldsymbol{r}}
$$

is illuminating the ground plane from the upper half-space, where $\hat{k}_{i}$ is the unit vector along the propagation direction given by

$$
\hat{k}_{i}=\hat{x} \sin \theta_{i} \cos \phi_{i}+\hat{y} \sin \theta_{i} \sin \phi_{i}-\hat{z} \cos \theta_{i} .
$$

The vector $\boldsymbol{E}_{\mathbf{0}}^{\boldsymbol{i}}$ in (1) is expressed in terms of a local coordinate system $\left(\hat{v}_{i}, \hat{h}_{i}, \hat{k}_{i}\right)$ where $\hat{h}_{i}=\hat{k}_{i} \times \hat{z} /\left|\hat{k}_{i} \times \hat{z}\right|$ and $\hat{v}_{i}=\hat{h}_{i} \times$ $\hat{k}_{i}$ denote the horizontal and vertical unit vectors, respectively. Representing the direction of the observation point by $\hat{k}_{s}$, the polarization of the scattered field can also be expressed in terms of a local coordinated system $\left(\hat{v}_{s}, \hat{h}_{s}, \hat{k}_{s}\right)$ where

$$
\hat{k}_{s}=\hat{x} \sin \theta_{s} \cos \phi_{s}+\hat{y} \sin \theta_{s} \sin \phi_{s}+\hat{z} \cos \theta_{s},
$$

and $\hat{v}_{s}$ and $\hat{h}_{s}$ can be obtained using similar expressions as those given for $\hat{v}_{i}$ and $\hat{h}_{i}$, respectively.

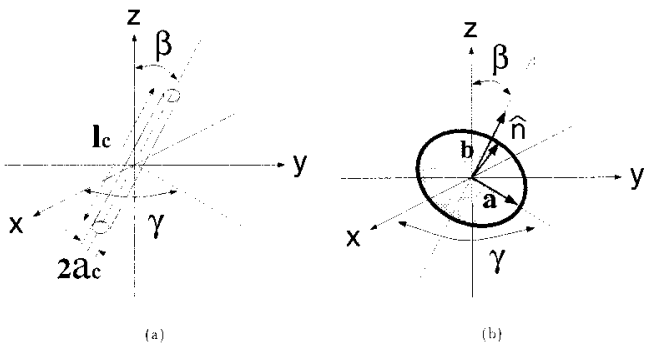

Fig. 2. Denotation of the dimensional and orientational parameters for (a) cylinder and (b) disk.

\section{A. Vegetation Structure Modeling}

To make the proposed scattering solution tractable, simple geometries are chosen to represent vegetation particles. Leaves are represented by elliptical thin dielectric disks. The other particles, which include stems, branches, and pods, are modeled using circular cylinders. Analytical scattering solutions are available for both geometries and will be introduced in the next section.

The orientation and dimension of each particle are described by four parameters, as shown in Fig. 2. The values of these parameters are determined by random number generators during the simulation with predescribed probability distribution functions (p.d.f.'s). The orientation parameters of the particles are described by two angles: $\beta$ (elevation angle) and $\gamma$ (azimuth angle). Azimuthal symmetry is assumed for $\gamma$, and its p.d.f. is given by

$$
p(\gamma)=\frac{1}{2 \pi}, \quad \gamma \in[0,2 \pi) .
$$

However, for $\beta$, a bell-shaped p.d.f. is chosen

$$
p(\beta)=\frac{e^{-\left(\left(\beta-\beta_{m}\right) / \beta_{s}\right)^{2}}}{\int_{0}^{\pi} e^{-\left(\left(\beta^{\prime}-\beta_{m}\right) / \beta_{s}\right)^{2}} d \beta^{\prime}}, \quad \beta \in[0, \pi] .
$$

For leaves, the axis ratio $b / a$ is assumed to be constant, and the thickness and major axis $a$ are given Gaussian p.d.f.'s. Three types of cylinders are considered for main stems, branches, and pods. For these cylinders, Gaussian p.d.f.'s are chosen to describe the statistics of their radii and lengths.

The branching structure of soybeans is rather simple and can be developed using the following algorithm.

1) All parameters of the main stem are determined using random number generators. The main stem is then divided into subsections, whose lengths are again decided by a Gaussian random number generator.

2) At each node (connecting point of two subsections of the stem), a branch is placed whose orientation is obtained from (4) and (5). Depending on the growth stage, pods may be added at each node.

3) To each branch end, a leaf is attached. In this paper, the number of leaflets at each branch end is three (this may be different for other soybean species). Azimuthal orientation angle of leaves is determined from the orientation angle of the branches to which they are connected. 


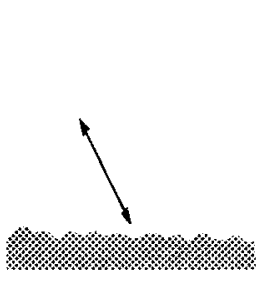

(a)

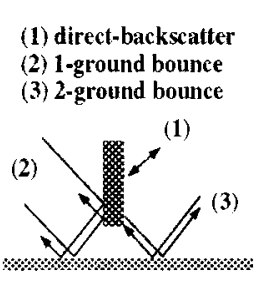

(b)

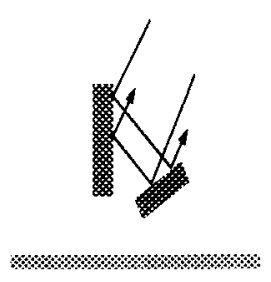

(c)

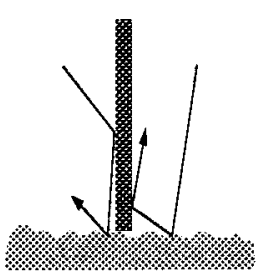

(d)

Fig. 3. Scattering mechanisms: (a) direct backscatter from rough surface, (b) direct backscatter from vegetation, single ground-bounce, and double ground-bounce, (c) second-order near-field interaction, and (d) incoherent main stem, rough surface interaction.

Fig. 9 shows a typical computer-generated soybean structure according to the aforementioned algorithm.

\section{B. Scattering Mechanism and Scattering Formulations for the Vegetation Particles and Rough Surfaces}

Several scattering mechanisms are considered for the scattering model. Fig. 3 depicts six different mechanisms including:

1) direct backscatter from the underlying rough surface;

2) direct backscatter from vegetation particles;

3) single ground bounce;

4) double ground bounce;

5) second-order scattering interaction among vegetation particles;

6) scattering interaction between main stem and the rough surface.

The first four mechanisms are included in almost all existing vegetation scattering models. Mechanism 5 is a second-order solution that accounts for the near-field interaction within a single plant. Mechanism 6 is only considered for predicting the cross-polarized scattering at L-band according to a study reported in [10], where it is shown that the copolarized scattering of mechanism 6 at L-band is weak compared to that of mechanism 2. Mechanism 6 is also ignored at C-band, because of attenuation experienced by the wave propagating through the vegetation layer. In what follows, the scattering solutions for each mechanism are briefly described.

Mechanism 1: There exist many rough-surface scattering models available in the literature. In this paper, a second-order small perturbation model (SPM) [17] and a physical optic (PO) model [18] are incorporated to handle the backscatter from the rough surface.

Mechanisms 2-4: These mechanisms are often referred to as the single scattering solutions, in which only the scattering solutions for the isolated vegetation particles are considered. The effect of the ground surface in mechanisms 3 and 4 are considered by introducing the ground reflection coefficients. If the SPM is used in mechanism 1, the Fresnel reflection coefficients are used directly. If the PO is needed according to the surface roughness condition, the reflection coefficients are modified by $e^{-2\left(k s \cos \theta_{i}\right)^{2}}$ to account for the reduction in the surface reflectivity [11]. The single scattering solutions for dielectric disks and cylinders are obtained from the following formulations:

Elliptical Disk: The thickness of the soybean leaves $\approx 0.2-0.3 \mathrm{~mm}$ is usually small compared to the wavelength in the microwave region, and the ratio of the thickness to the diameter of the leaves is much less than unity. Also, by noting that the dielectric constant of vegetation is lossy, the Rayleigh-Gans formulation [12] can be applied to derive the scattering solution for the elliptical disks representing the vegetation leaves. For an elliptical disk, the scattering matrix elements are found to be

$$
S_{p q}^{d}=\hat{p} \cdot\left(\overline{\boldsymbol{P}}_{d} \cdot \hat{q}\right) \frac{A_{d} k_{0}^{2}}{2 \pi} \frac{J_{1}\left(\sqrt{(a A)^{2}+(b B)^{2}}\right)}{\sqrt{(a A)^{2}+(b B)^{2}}}
$$

where

$A_{d} \quad$ area axis of the disk;

a major axis of the disk;

$b \quad$ minor axis of the disk.

In (6), $\overline{\bar{P}}_{\boldsymbol{d}}=\overline{\bar{U}}_{\boldsymbol{d}}^{-1} \overline{\overline{\boldsymbol{P}}}_{\boldsymbol{d}}^{0} \overline{\bar{U}}_{\boldsymbol{d}}$, where $\overline{\bar{P}}_{\boldsymbol{d}}^{0}$ is the disk's polarizability tensor, which can be found in [12] and [13], and $\overline{\overline{U_{\boldsymbol{d}}}}$ is the matrix of the coordinate transformation that transfers the global coordinate system to a local coordinate system defined by the major axis, minor axis, and the normal of the disk, respectively. The explicit expression for $\overline{\overline{\boldsymbol{U}_{\boldsymbol{d}}}}$ can be obtained from [14]. Also, $A$ and $B$ are given by

$$
\begin{aligned}
& A=k_{0}\left[\overline{\overline{\boldsymbol{U}}} \overline{\boldsymbol{d}}^{\mathbf{1}} \cdot\left(\hat{k}_{i}-\hat{k}_{s}\right)\right] \cdot \hat{x} \\
& B=k_{0}\left[\overline{\overline{\boldsymbol{U}}}-\mathbf{d} \cdot\left(\hat{k}_{i}-\hat{k}_{s}\right)\right] \cdot \hat{y} .
\end{aligned}
$$

Circular Cylinder: An exact scattering solution does not exist for cylinders of finite length, but an approximated solution, assuming the internal field induced within the finite cylinder, is the same as that of the infinite cylinder with the same cross section and dielectric constant, and can be used effectively [15]. Generally, this solution is valid when the ratio of the length to the diameter is large.

Mechanism 5: The second-order scattered field between two particles is formulated using an efficient algorithm based on the reciprocity theorem [7]. For two adjacent particles, we have

$$
\hat{p} \cdot \boldsymbol{E}_{\mathbf{2 1}}=\int_{V_{1}} \boldsymbol{E}_{e 2} \cdot \boldsymbol{J}_{1} d v .
$$

where $\boldsymbol{E}_{e 2}$ is the scattered field from particle \#2 illuminated by an infinitesimal current source at the observation point in the absence of particle \#1, and $\boldsymbol{J}_{1}$ is the induced polarization current of particle \#1 illuminated by the incidence field in the absence of particle \#2. $\boldsymbol{E}_{12}$ can be obtained using the reciprocity theorem. Hence the second-order scattered field are conveniently 
obtained from the plane wave solution of the induced polarization current and near field of individual particles. These quantities for disks and cylinders are given by:

1) Disk: The induced polarization current is obtained from the Rayleigh-Gans approximation and is given by

$$
J_{1}(\boldsymbol{r})=-i k_{\circ} Y_{o} \overline{\bar{P}}_{\boldsymbol{d}} \cdot \boldsymbol{E}_{\mathbf{0}}^{\boldsymbol{i}} e^{i k_{0} \hat{k}_{i} \cdot \boldsymbol{r}}
$$

where $\overline{\bar{P}}_{\boldsymbol{d}}$ is the polarizability tensor. The exact near-field scattered field must be numerically evaluated from

$$
\begin{aligned}
\boldsymbol{E}_{e 2}(\boldsymbol{r})= & \frac{i k_{0} Z_{0}}{(4 \pi)^{2}} \frac{e^{i k_{0} r_{0}}}{r_{0}}\left(\overline{\overline{\boldsymbol{P}}}_{\boldsymbol{d}} \cdot \hat{p}\right) \\
& \cdot \int_{S_{2}} \overline{\bar{G}}(k, R) e^{i k_{0}\left(-\hat{k}_{s} \cdot \boldsymbol{r}^{\prime}+R\right)} d s^{\prime}
\end{aligned}
$$

where

$$
\begin{aligned}
\overline{\overline{\boldsymbol{G}}}\left(k_{0}, R\right)= & \left(\frac{-1+i k_{0} R+k_{0}^{2} R^{2}}{R^{3}}\right) \overline{\overline{\boldsymbol{I}}} \\
& +\left(\frac{3-3 i k_{0} R-k_{0}^{2} R^{2}}{R^{3}}\right) \hat{R} \hat{R}
\end{aligned}
$$

and $\hat{R}$ is a unit vector defined by $\hat{R}=\left(\boldsymbol{r}-\boldsymbol{r}^{\prime}\right) /\left|\boldsymbol{r}-\boldsymbol{r}^{\prime}\right|$.

2) Cylinder: The formulation for finite cylinders is used again to calculate the induced polarization current and the near-field scattered field. The formulation of the scattered field in the vicinity of the cylinder is given by [7]

$$
\begin{aligned}
\boldsymbol{E}_{e 2}(\boldsymbol{r})= & -\frac{i k_{o} Z_{o} e^{i k_{o} r_{o}}}{4 \pi r_{o}} \boldsymbol{F}\left(\phi-\phi_{s}\right) H_{o}^{(1)} \\
& \cdot\left(k_{o} \sin \theta_{s} \rho\right) e^{k_{o} \cos \theta_{s} z} .
\end{aligned}
$$

Equation (12) is derived using the stationary phase approximation along the axial direction of the cylinder axis. This solution has been verified by the method of moments (MoM) [7], [16], and the region of validity is given by

$$
\rho>2 d_{c}^{2} / \lambda
$$

where $d_{c}$ is the diameter of the cylinder, and $\rho$ is the radial distance between the observation point and the cylinder axis. For the main stem of soybeans, the radius is usually less than $5 \mathrm{~mm}$. Applying (13), it is found that $\rho>3.5$ $\mathrm{mm}$ at C-band (5.3 GHz). Therefore, (12) is appropriate for calculating the near-field interaction.

Mechanism 6: The incoherent interaction between the main stems and rough surface is formulated using the reciprocity technique introduced in [7]. The details and lengthy formulation for the cylinder-rough surface scattering interaction can be found in [19]. This model is only applied to calculate the scattering interaction between the main stem and underlying rough surface. The reason for this is that for a titled cylinder with large elevation angle $\beta$ (such as branches), the cross-polarized scattering from mechanisms 2 and 3 is dominant. However, main stems often grow nearly vertically, and its interaction with the ground becomes an important source of the cross-polarized scattering, noting that the mechanisms 2 and 3 of nearly vertical

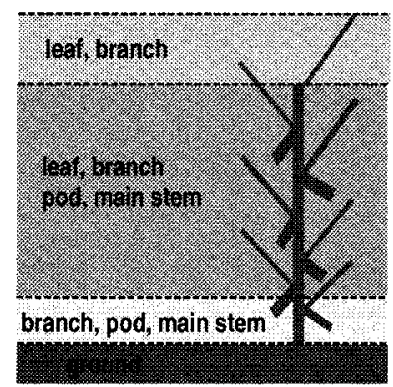

(a)

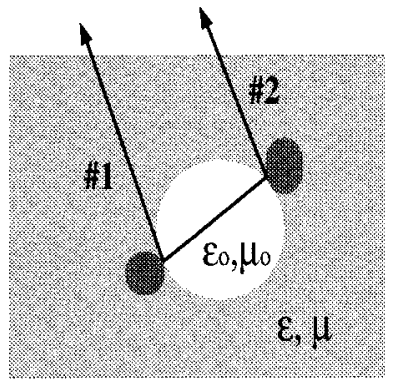

(b)
Fig. 4. Vegetation particles embedded in the lossy medium: (a) stratified structure for the calculation of the equivalent propagation constant and (b) free space is assumed in the calculation of the second-order near-field interaction.

cylinders do not produce significant cross-polarized scattering fields. As will be shown later, the cross-polarized scattering at L-band is mainly dominated by two scattering mechanisms 2 and 6.

\section{Propagation in a Lossy Layered Media}

1) Foldy's Approximation: The scattering solutions provided in the previous section are for targets in free space. However, for vegetation canopies the targets are within a lossy random medium. Thus, a particle is illuminated by not only the incident plane wave, but also by the scattered fields from other particles. To calculate the total scattered field from a particle, it is usually assumed that the particle is embedded in a homogeneous lossy medium, as shown in Fig. 4(a). The vegetation layer can be divided into many sublayers that contain different types and number densities of vegetation particles and thus, each layer exhibits different equivalent propagation constants. Foldy's approximation [14] has been widely used in many vegetation scattering models to account for the attenuation experienced by the wave traveling through the vegetation medium. According to the Foldy's approximation, the vertical and horizontal components of the mean electric field in a sparse random medium satisfy

$$
\begin{aligned}
& \frac{d E_{h}}{d s}=i\left(k_{0}+M_{h h}\right) E_{h}+i M_{h v} E_{v} \\
& \frac{d E_{v}}{d s}=i M_{v h} E_{h}+i\left(k_{0}+M_{v v}\right) E_{v}
\end{aligned}
$$

where $s$ is the length along the propagation path within the medium, and

$$
M_{p q}=\frac{2 \pi n_{0}}{k_{0}}\left\langle S_{p q}(\hat{k}, \hat{k})\right\rangle, \quad p, q \in\{h, v\} .
$$

Here, $n_{0}$ is the number density of the scatterers within the medium, and $\left\langle S_{p q}(\hat{k}, \hat{k})\right\rangle$ is the averaged forward scattering matrix element of the scatterers. Since the vegetation structure exhibits statistical azimuthal symmetry, there is no coupling between horizontal and vertical components of the coherent field and therefore, $M_{h v}=M_{v h}=0$. From (14), the effective propagation constants for both polarizations are given by

$$
\begin{aligned}
& k_{h}^{e}=k_{0}+M_{h h} \\
& k_{v}^{e}=k_{0}+M_{v v} .
\end{aligned}
$$




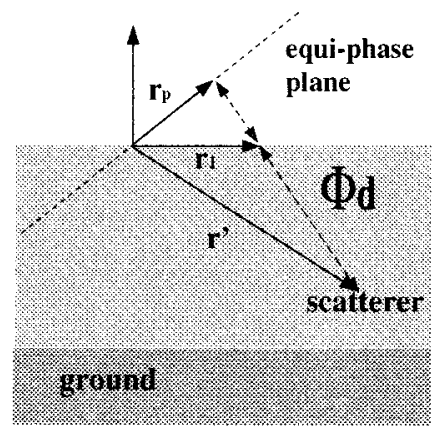

(a)

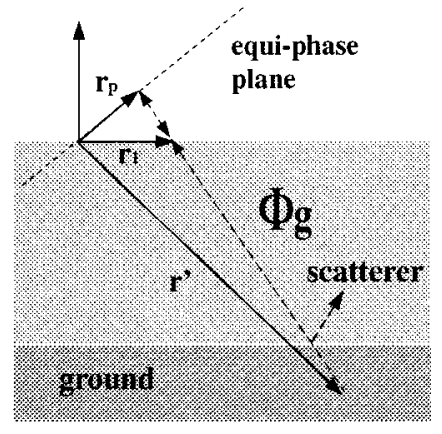

(b)
Fig. 5. Propagation paths in the vegetation layer: (a) direct and (b) ground bounce.

As mentioned previously, the second-order near-field interaction is incorporated in this model, and it will only be calculated for the scatterers within a single plant. It is reasonable to assume that no extinction should be considered for the calculation of the near-field interaction. However, since both particle are still embedded in the vegetation layer, extinction is considered for the incident wave and secondary scattered fields. As shown in Fig. 4(b), the space between two scatterers is considered as free space, and Foldy's approximation is still used on paths 1 and 2.

2) Propagation Paths: In this section, the phase difference and extinction caused by the wave propagating in the vegetation layer will be formulated using the method presented in [20]. To build a coherent scattering model, the phase of each scattering mechanism has to be calculated with respect to a phase reference point. Fig. 5(a) shows the propagation geometry for the direct path. The reference phase point is taken to be the origin of the coordinate system. Using ray optics, the propagation from the equi-phase plane [shown in Fig. 5(a)] directly to the scatterer is given by

$$
\Phi_{d}^{\prime}\left(\hat{k}_{0}, \boldsymbol{r}^{\prime}, p\right)=k_{0} \boldsymbol{r}_{1} \cdot \hat{k}_{0}+k_{p}^{e}\left(\boldsymbol{r}^{\prime}-\boldsymbol{r}_{1}\right) \cdot \hat{k}_{e}
$$

where $\boldsymbol{r}_{1}$ denotes the location at which the ray intersects the interface between the vegetation layer and free-space. Here, the effect of refraction is ignored assuming a diffuse boundary between the vegetation layer and free-space $\hat{k}_{e}=\hat{k}_{0}$, and $p$ denotes the polarization of the wave. Substituting (16) into (17), it is found that

$$
\boldsymbol{\Phi}_{d}^{\prime}\left(\hat{k}_{0}, \boldsymbol{r}^{\prime}, p\right)=k_{0} \boldsymbol{r}^{\prime} \cdot \hat{k}_{0}+M_{p p}\left(\boldsymbol{r}^{\prime}-\boldsymbol{r}_{\mathbf{1}}\right) \cdot \hat{k}_{0} .
$$

The first term on the right-hand side of (18) is the free-space propagation term and will be included in the scattering matrix elements of the scatterer. The second term on the right-hand side is the extra phase difference and extinction caused by the propagation in the lossy vegetation media, and will be denoted as $\boldsymbol{\Phi}_{d}\left(\hat{k}_{0}, \boldsymbol{r}^{\prime}, p\right)$. The free space-vegetation interface is set to be the $x-y$ plane, so it is found that

$$
\left(\boldsymbol{r}^{\prime}-\boldsymbol{r}_{\mathbf{1}}\right) \cdot \hat{k}_{0}=\frac{z^{\prime}}{\hat{k}_{o} \cdot \hat{z}}
$$

TABLE I

MEASURED GROUND TRUTH FOR THE POLARSCAT DATA SET

\begin{tabular}{c|c|c}
\hline & Aug. 14 & Aug. 18 \\
\hline soil $\left(m_{v}\right)$ & 0.06 & 0.17 \\
\hline rms height $(s)$ & \multicolumn{2}{|c}{$0.0115 \mathrm{~m}$} \\
\hline correlation length $(l)$ & \multicolumn{2}{|c}{$0.0879 \mathrm{~m}$} \\
\hline vegetation $\left(m_{g}\right)$ & 0.769 & 0.767 \\
\hline number density of plant & $34 \pm 13 \mathrm{plants} / \mathrm{m}^{2}$ \\
\hline biomass & \multicolumn{2}{|c|}{$1.97 \mathrm{~kg} / \mathrm{m}^{2}$} \\
\hline
\end{tabular}

Therefore, $\boldsymbol{\Phi}_{d}\left(\boldsymbol{r}^{\prime}, p\right)$ can be written as

$$
\Phi_{d}\left(\hat{k}_{0}, \boldsymbol{r}^{\prime}, p\right)=M_{p p} \frac{z^{\prime}}{\hat{k}_{o} \cdot \hat{z}} .
$$

The ground-bounce path, as shown in Fig. 5(b), includes a reflection from the ground plane. In Fig. 5(b), the image position is given by

$$
\boldsymbol{r}_{\text {image }}^{\prime}=x^{\prime} \hat{x}+y^{\prime} \hat{y}-\left(z^{\prime}+2 d\right) \hat{z}
$$

where $d$ is the thickness of the layer. Using (20), it is found that $\Phi_{g}\left(\hat{k}_{0}, r^{\prime}, p\right)$, which only accounts for the extra phase difference and extinction caused by the propagation in the lossy vegetation media, can be written as

$$
\boldsymbol{\Phi}_{g}\left(\hat{k}_{0}, \boldsymbol{r}^{\prime}, p\right)=-M_{p p} \frac{z^{\prime}+2 d}{\hat{k}_{o} \cdot \hat{z}} .
$$

\section{Scattering from Soybean Fields and Monte Carlo Simulation}

Consider an area of soybean field with $N_{p}$ soybean plants per unit area. For a given computer-generated soybean plant (the $k$ th plant with $N_{s}$ particles), the total scattering amplitude can be written as

$$
\begin{aligned}
S_{p q, k}=\left\{\sum_{i=1}^{N_{s}}\left[S_{p q, k i}^{d}+S_{p q, k i}^{g g}+S_{p q, k i}^{g 1}+S_{p q, i}^{g 2}\right]\right. \\
\left.+\sum_{i=1}^{N_{s}} \sum_{\substack{j=1 \\
j \neq i}}^{N_{s}} S_{p q, k i j}^{2 n d}\right\} e^{i k_{0}\left(\hat{k}_{i}-\hat{k}_{s}\right) \cdot \boldsymbol{r}_{\boldsymbol{k}}}
\end{aligned}
$$

where $\boldsymbol{r}_{\boldsymbol{k}}$ is the location of the plant. In (23), each term includes the attenuation and phase shift due to the propagation

direct:

$$
S_{p q, k i}^{d}=S_{p q, k i}\left(\hat{k}_{s}, \hat{k}_{i}\right) e^{i \Phi_{d}\left(-\hat{k}_{s}, \boldsymbol{r}_{k i}, p\right)} e^{i \Phi_{d}\left(\hat{k}_{i}, \boldsymbol{r}_{k i}, q\right)}
$$

ground-plant:

$$
S_{p q, k i}^{g 1}=S_{p q, k i}\left(\hat{k}_{s}, \hat{k}_{i}^{\prime}\right) R_{p} e^{i \Phi_{g}\left(-\hat{k}_{s}, \boldsymbol{r}_{k i}, p\right)} e^{i \Phi_{d}\left(\hat{k}_{i}, \boldsymbol{r}_{k i}, q\right)}
$$

plant-ground:

$$
S_{p q, k i}^{g 1}=S_{p q, k i}\left(\hat{k}_{s}^{\prime}, \hat{k}_{i}\right) R_{q} e^{i \Phi_{d}\left(-\hat{k}_{s}, \boldsymbol{r}_{k i}, p\right)} e^{i \Phi_{g}\left(\hat{k}_{i}, \boldsymbol{r}_{k i}, q\right)}
$$

ground-ground:

$$
S_{p q, k i}^{g g}=S_{p q, k i}\left(\hat{k}_{s}^{\prime}, \hat{k}_{i}^{\prime}\right) R_{q} R_{q} e^{i \Phi_{g}\left(-\hat{k}_{s}, \boldsymbol{r}_{k i}, p\right)} e^{i \Phi_{g}\left(\hat{k}_{i}, \boldsymbol{r}_{k i}, q\right)}
$$

near-field 2nd-order:

$$
S_{p q, k i j}^{2 n d}=S_{p q, k i j}^{2 n d}\left(\hat{k}_{s}, \hat{k}_{i}\right) e^{i \Phi_{d}\left(-\hat{k}_{s}, \boldsymbol{r}_{k i}, p\right)} e^{i \Phi_{d}\left(\hat{k}_{i}, \boldsymbol{r}_{k, j}, q\right)}
$$

where $\hat{k}_{i}^{\prime}=\hat{k}_{i}-2\left(\hat{k}_{i} \cdot \hat{z}\right) \hat{z}$ and $\hat{k}_{s}^{\prime}=\hat{k}_{s}-2\left(\hat{k}_{s} \cdot \hat{z}\right) \hat{z}$. Note that all scattering mechanisms are added coherently to capture the coherence effect caused by the vegetation structure. 


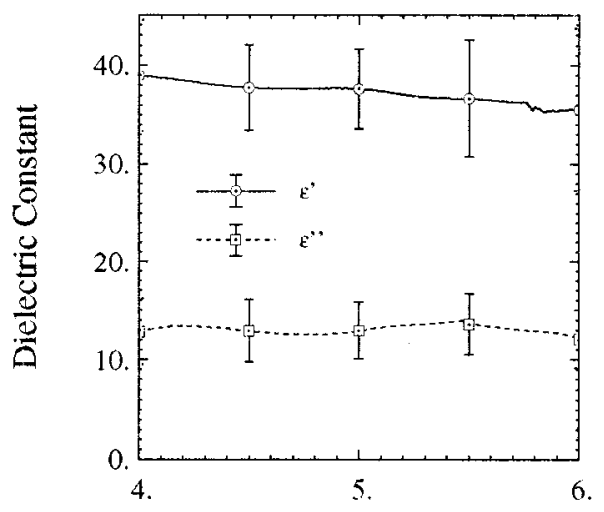

Frequency $(\mathrm{GHz})$

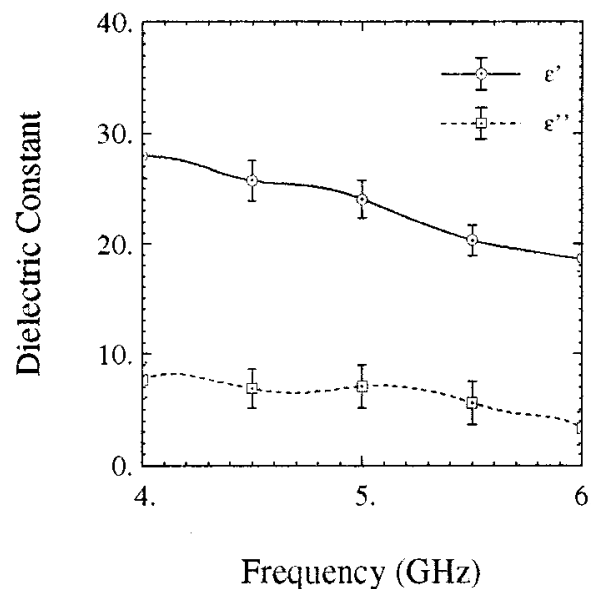

(b)

Fig. 6. Measured dielectric constants for (a) branches and main stems and (b) leaves at C-band using the procedure outlined in [24] and [25].

The scattering coefficient of the soybean field is then computed by incoherent addition of the scattered powers from vegetation, rough surface, and main stem-rough surface interaction. Hence

$$
\begin{aligned}
\sigma_{p q p q}^{0}= & \sigma_{p q p q}^{0}(\text { vegetation })+\sigma_{p q p q}^{0}(\text { rough surace }) \\
& +\sigma_{p q p q}^{0}(\text { stem-rough surface })
\end{aligned}
$$

where

$$
\begin{aligned}
& \sigma_{p q p q}^{0}(\text { vegetation })=4 \pi\left\langle\left|\sum_{k=1}^{N_{p}} S_{p q, k}\right|^{2}\right\rangle \\
& \sigma_{p q p q}^{0}(\text { rough surface }) \\
& \quad=\sigma_{p q p q, r}^{0}\left|e^{i \Phi_{d}\left(-\hat{k}_{s},-d \hat{z}, p\right)} e^{i \Phi_{d}\left(\hat{k}_{i},-d \hat{z}, q\right)}\right|^{2} \\
& \sigma_{p q p q}^{0}(\text { stem-rough surface }) \\
& \quad=4 \pi N_{p}\langle| S_{p q}^{r c} e^{i \Phi_{d}\left(-\hat{k}_{s},-d \hat{z}, p\right)} e^{i \Phi_{d}\left(\hat{k}_{i},\left(-d+0.5 l_{c}\right) \hat{z}, q\right)} \\
& \left.\quad+\left.S_{p q}^{c r} e^{i \Phi_{d}\left(\hat{k}_{i},-d \hat{z}, p\right)} e^{i \Phi_{d}\left(-\hat{k}_{s},\left(-d+0.5 l_{c}\right) \hat{z}, q\right)}\right|^{2}\right\rangle .
\end{aligned}
$$

In calculation of the contribution from the direct rough surface and the stem-rough surface, the propagation attenuation through vegetation layer is also included. $S_{p q}^{r c}$ and $S_{p q}^{c r}$ are, respectively, the rough surface cylinder and cylinder rough surface scattering amplitudes. The ensemble averaging in (28) is carried out analytically using the SPM formulation, and the details are reported in [10]. As mentioned earlier, the contribution from this term is only significant at L-band for the cross-polarized term.

The ensemble averaging in (28) is carried out using a Monte Carlo simulation. For each realization in the Monte Carlo simulation, a group of computer-generated soybean plants are generated and distributed on a square area of $1 \mathrm{~m}^{2}$, and then the scattered fields are computed. This procedure will be repeated until a convergence is reached. To examine the coherence effect, the scattered power from the vegetation is also calculated incoherently from

$$
\begin{aligned}
& \sigma_{p q p q}^{0}(\text { vegetation }) \\
& =4 \pi\left\langle\sum _ { k = 1 } ^ { N _ { p } } \left\{\sum _ { i = 1 } ^ { N _ { s } } \left[\left|S_{p q, k i}^{d}\right|^{2}+\left|S_{p q, k i}^{g g}\right|^{2}\right.\right.\right. \\
& \left.\left.\left.+\left|S_{p q, k i}^{g 1}\right|^{2}+\left|S_{p q, i}^{g 2}\right|^{2}\right]+\sum_{i=1}^{N_{s}} \sum_{\substack{j=1 \\
j \neq i}}^{N_{s}}\left|S_{p q, k i j}^{2 n d}\right|^{2}\right\}\right\rangle .
\end{aligned}
$$

\section{EXPERIMENTAL RESULTS}

In this section, the experimental procedure and the multifrequency multipolarization backscatter measurements using polarimetric scatterometer systems and JPL AIRSAR are presented.

\section{A. Measurement Using the University of Michigan's POLARSCAT}

In August 1995, a series of polarimetric measurements were conducted on a soybean field near Ann Arbor, MI. These measurements were conducted using the University of Michigan polarimetric scatterometer systems (POLARSCAT) [21]. The polarimetric backscatter data were collected at two different frequencies (L-band and C-band) over a wide range of incidence angles (from $20-70^{\circ}$ at $10^{\circ}$ increment). The overall goal of these experiments was to investigate the feasibility of soil-moisture retrieval of vegetation-covered terrain from radar backscatter data. Experiments were designed to observe the radar-backscatter variations due to the change in soil moisture, while the vegetation parameters were almost the same. Two sets of data were collected. In one measurement, the angular polarimetric data were collected on August 14, when the underlying soil surface was dry, and in another a similar data was collected right after a heavy rain on August 18. At the 
TABLE II

MEASUREd Vegetation PARAMETERS OF SOYBEANS For THE POLARSCAT DATA SET

\begin{tabular}{c|c|c|c}
\hline & $\beta_{m}, \beta_{s}$ (degree) & radius $(\mathrm{cm})$ & length or thickness $(\mathrm{cm})$ \\
\hline stem & 5,5 & $0.3 \pm 0.09$ & $73.0 \pm 3.4$ \\
\hline node & 5,5 & $0.3 \pm 0.09$ & $5.4 \pm 1.4$ \\
\hline branch & $45.8,25.6$ & $0.12 \pm 0.031$ & $20.7 \pm 6.5$ \\
\hline pod & $135.5,30.8$ & $0.35 \pm 0.03$ & $3.7 \pm 0.48$ \\
\hline leaf & $45.6,30.1$ & $3.8 \pm 0.07(0.576)$ & $0.022 \pm 0.002$ \\
\hline
\end{tabular}

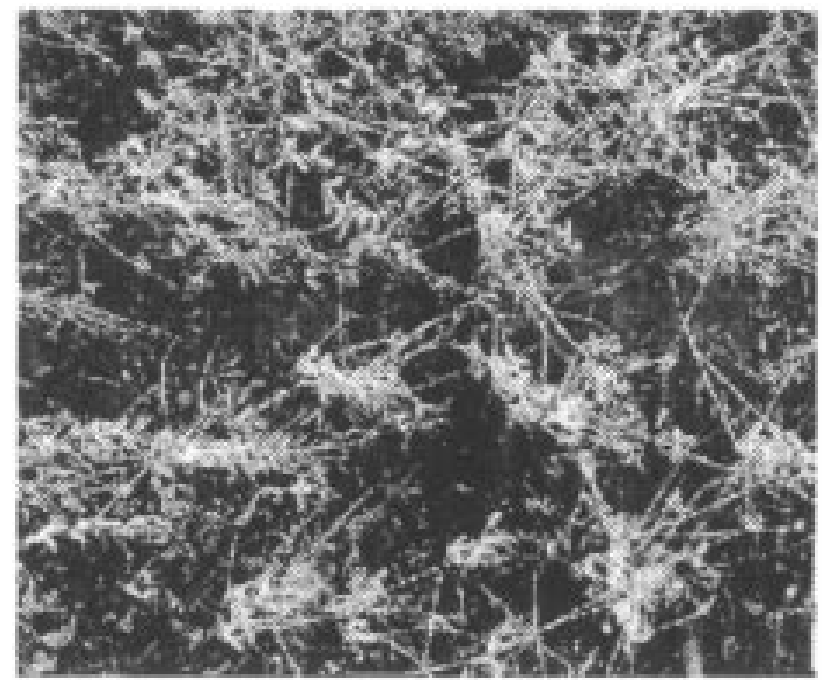

Fig. 7. Picture of the soybean plant distribution for POLARSCAT data set. It was taken from the top of the field when plants were dry. Unlike the row structure, which is often seen in many cultivated fields, the distribution pattern is rather random.

time of experiments the soybean plants were fully grown with significant numbers of pods. In fact, the vegetation biomass was at its maximum. Since the separation between the time of experiments was only about four days, no significant change in the vegetation parameters were observed.

The vegetation structural parameters and moisture in addition to the soil surface roughness and moisture were carefully characterized. The dielectric constant of the soil surface was measured by using a C-band field-portable dielectric probe [22]. The measured relative dielectric constant $\epsilon_{r}$ was used to estimate the moisture contents $m_{v}$ by inverting a semiempirical model [23], which gives $\epsilon_{r}$ in terms of $m_{v}$. The mean $m_{v}$, which is shown in Table I, is then used to estimate $\epsilon_{r}$ at L-band.

Two dielectric measurement techniques [24], [25] were used to measure the dielectric constant of leaves and stems. These measurements were performed at C-band using a WR-187 waveguide sample holder, and the results are shown in Fig. 6. The corresponding dielectric constants at L-band were then calculated using the empirical model provided in [26]. The gravimetric moisture content $m_{g}$ of the vegetation was also measured on the day of radar measurement to monitor the variation of the biomass. As shown in Table I, the vegetation moisture remained almost the same on both dates of the experiments.

The dimensions and orientations of vegetation particles were also recorded. Table II shows the means and standard deviations of vegetation parameters. Unlike most cultivated fields, where

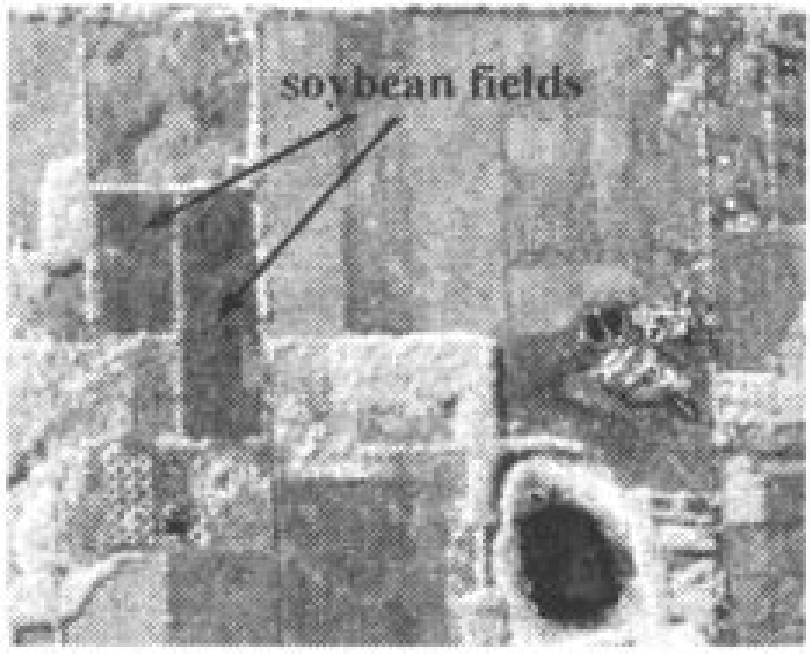

Fig. 8. AIRSAR image of the Kellogg Biological Station in July of 1995. This image combined the L-band and C-band backscatter data at $45^{\circ}$ of incidence angle. Two soybean fields are on the left side of the image with dark color.

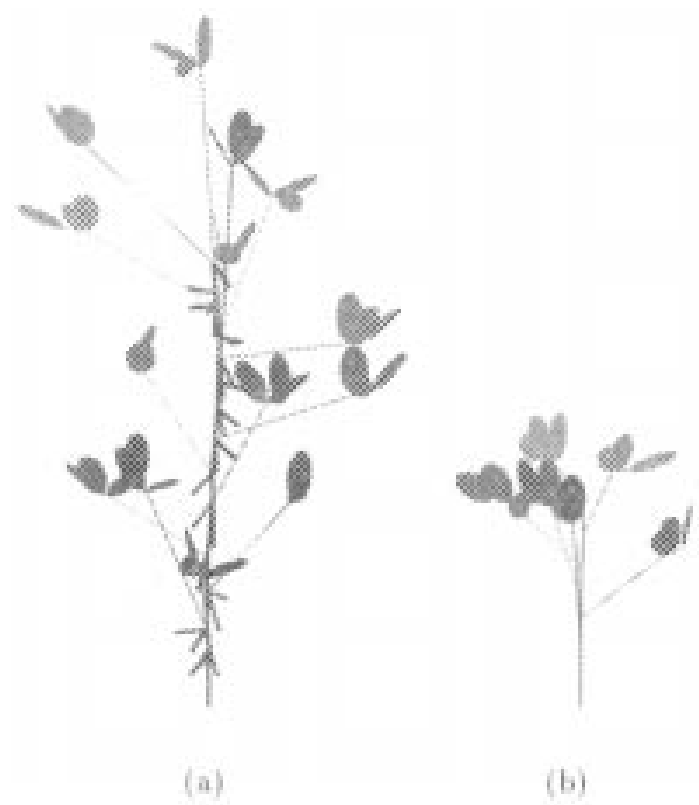

Fig. 9. Computer-generated soybean plants for: (a) POLARSCAT data set and (b) AIRSAR data set.

the plants are planted in row structures, the soybean plants of this field were distributed in a rather random pattern, as shown in Fig. 7. This picture shows the top-view at the end of the season, where all the leaves were fallen. The surface roughness parameters were also measured and reported in Table I.

\section{B. Measurement Using AIRSAR}

The Jet Propulsion Laboratory's airborne synthetic aperture radar (AIRSAR) [27] was deployed to conduct backscatter measurements on a number of cultivated fields. Although AIRSAR is capable of measuring polarimetric backscatter at three microwave frequencies (P-band, L-band, and C-band), only L-band and C-band data were collected. The backscatter data were collected by AIRSAR during its flight over the Kellogg Biological Station near Kalamazoo, MI, on July 12, 


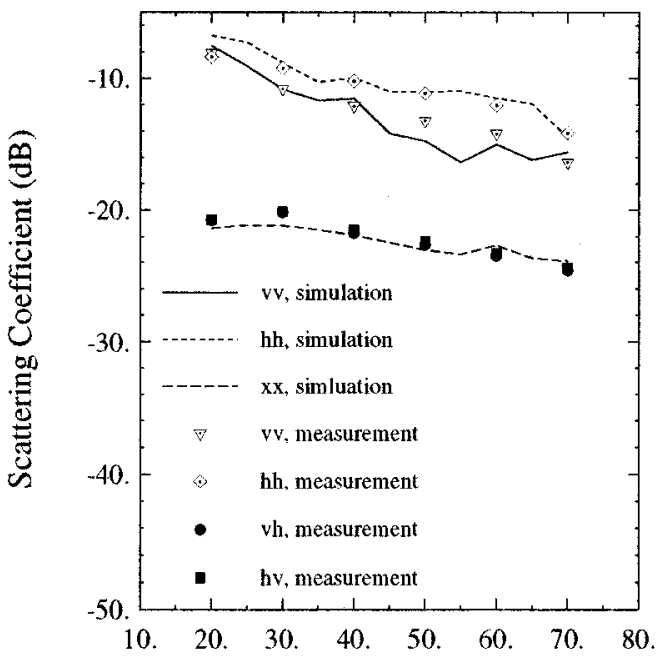

Incidence Angle (degree)

(a)

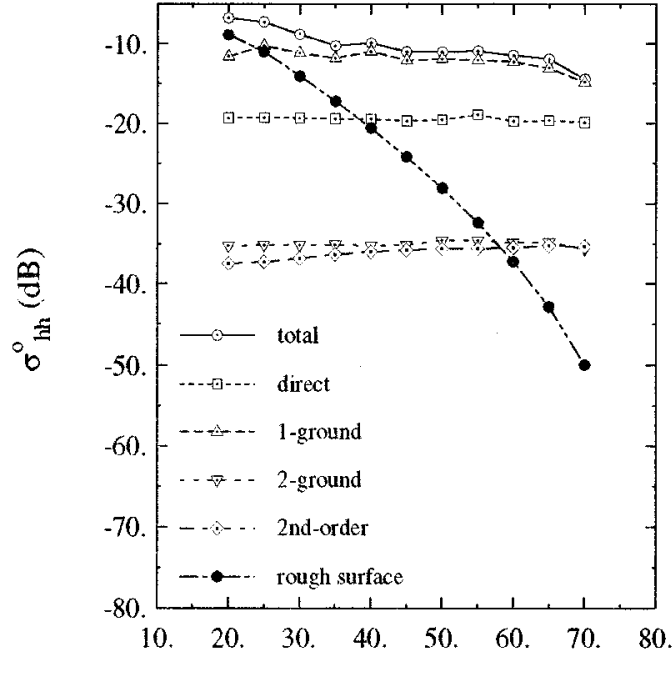

Incidence Angle (degree)

(c)

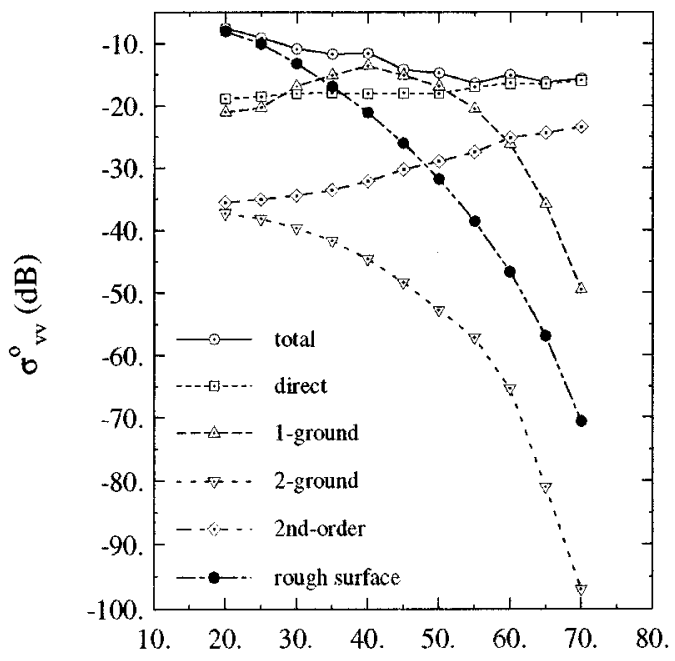

Incidence Angle (degree)

(b)

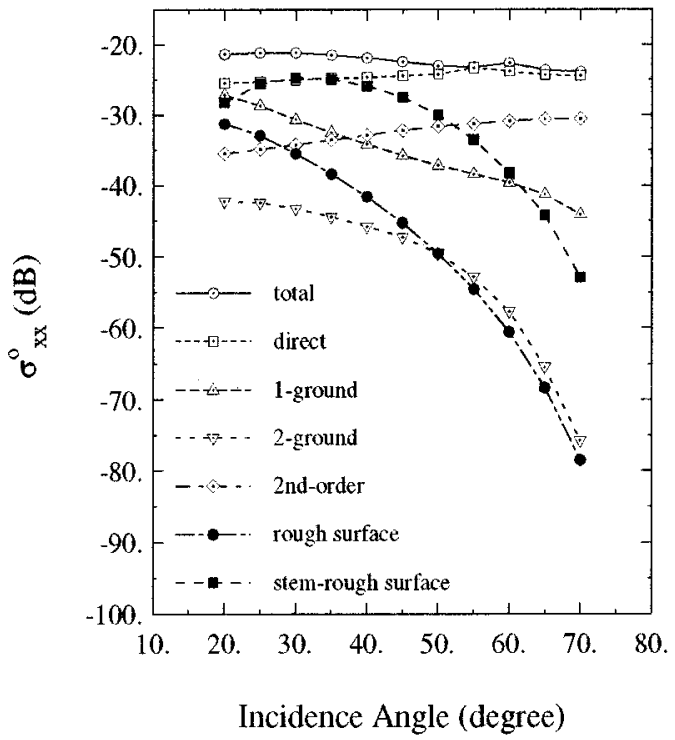

(d)

Fig. 10. Scattering coefficients versus incidence angle at L-band for August 14 POLARSCAT dataset: (a) model validation and scattering mechanism analysis for (b) vv-polarizations, (c) hh-polarizations, and (d) cross-polarizations.

1995. Also these data sets were collected at three different incidence angles: $30^{\circ}, 40^{\circ}$, and $45^{\circ}$. Unfortunately, the soybean fields were not within the research site of the station and the ground truth data was rather limited. The only available information is that the soybeans were about a month old and the volumetric soil moisture content was less than 0.1. Fig. 8 shows the composite L-band and C-band SAR image at $45^{\circ}$ incidence angle.

\section{Data Simulation and ANalysis}

The vegetation scattering model is first validated using the data collected by POLARSCAT. Guided by the ground truth data, many soybean plant structures were generated in order to carry on the data simulation [see Fig. 9(a)]. The computer-generated plants were uniformly distributed using a random number generator. The Monte Carlo simulations are performed at incidence angles ranging from $20-70^{\circ}$ at $5^{\circ}$ increment. Figs. 10(a) and 11(a) show the simulated and measured backscattering coefficients versus incidence angle at L-band and C-band, respectively. Good agreement is achieved by allowing the dielectric constants of vegetation particles to vary within the confidence region shown in Fig. 6. In Fig. 10(b)-(d), the contributions from individual scattering mechanisms are plotted as functions of incidence angle at L-band. The cross products among different mechanisms, which account for the coherence effect, are not presented in these figures. It is quite obvious that the contribution from the 


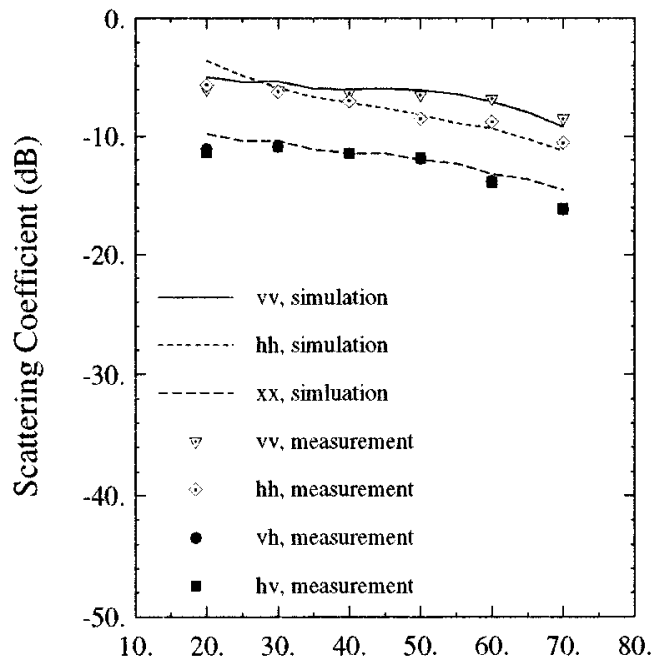

Incidence Angle (degree)

(a)

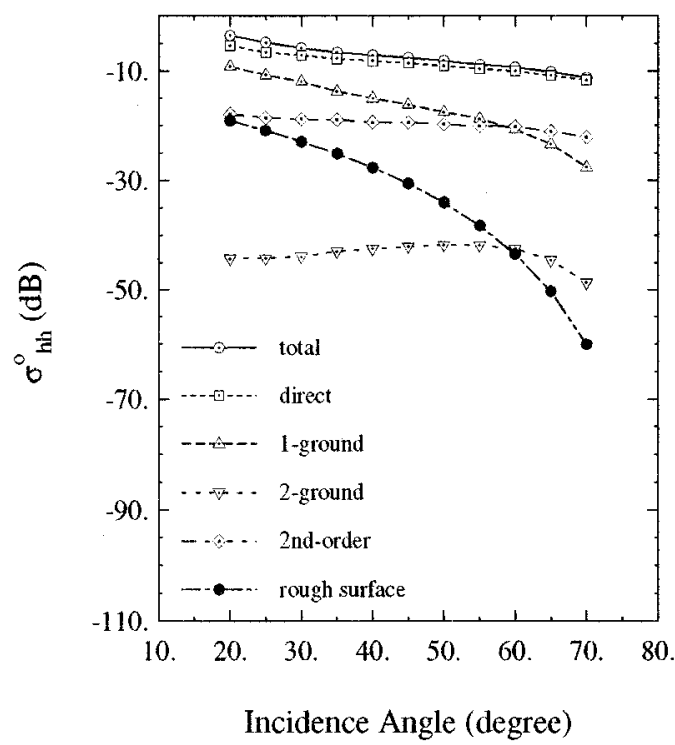

(c)

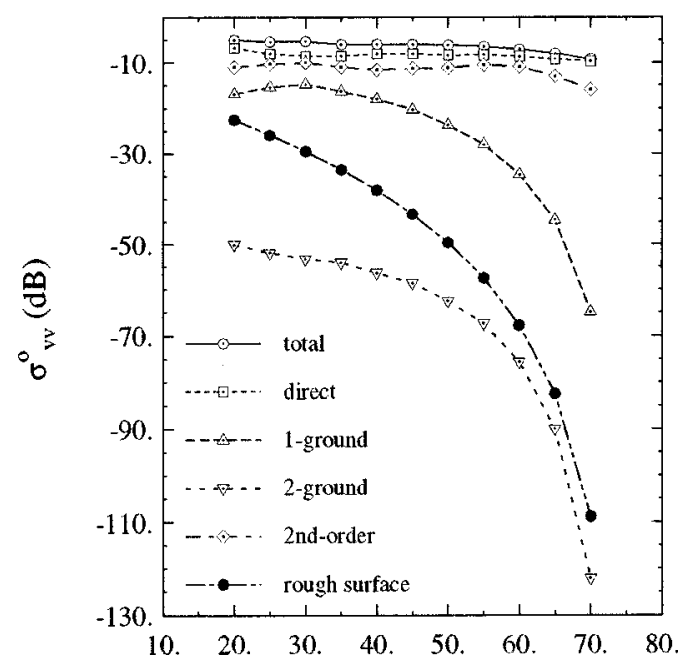

Incidence Angle (degree)

(b)

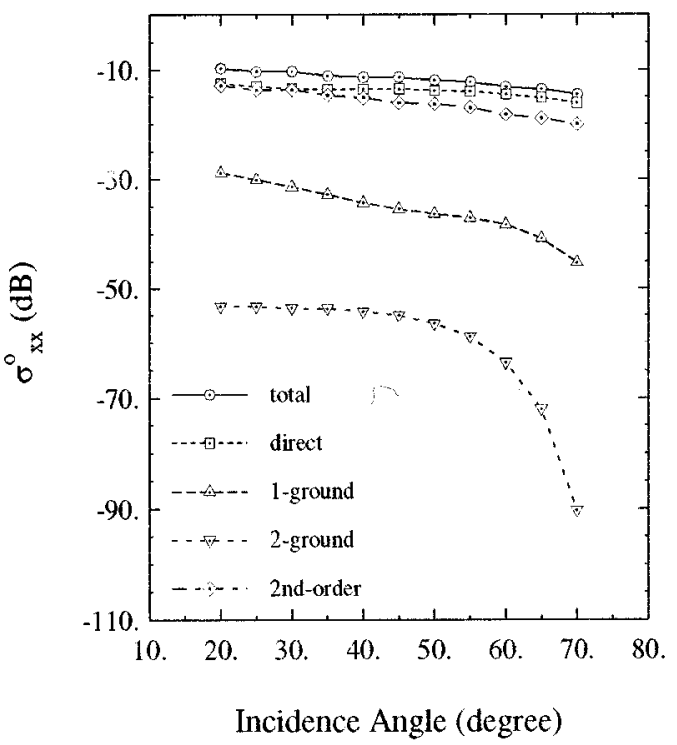

(d)

Fig. 11. Scattering coefficients versus incidence angle at C-band for August 14 POLARSCAT dataset: (a) model validation and scattering mechanism analysis for (v) vv-polarizations, (c) hh-polarizations, and (d) cross-polarizations.

second-order near-field interaction at L-band is negligible for both co- and cross-polarized terms. It is also shown that for copolarized backscattering coefficient, the direct backscatter from soybean, direct backscatter from rough surface, and single ground-bounce are sufficient to characterize the scattering behavior. For cross-polarization, however, the two most significant mechanisms are the direct backscatter from vegetation and the incoherent rough surface-stem interaction. The later mechanism contains information regarding the underlying soil surface including the soil moisture. Fig. 11(b)-(d) shows scattering contributions from different mechanisms versus incidence angle at $\mathrm{C}$-band. The direct backscatter form vegetation and the second-order near-field interaction are the dominant scattering mechanisms at C-band. Because of larger near-field region, the near-field interaction is stronger at $\mathrm{C}$-band than at L-band. Also the second-order near-field interaction has a more profound effect on the vv- and cross-polarization, because the orientation of the main stems is nearly vertical. The other mechanisms, which include the soil moisture information, are not significant for two reasons. 1) high extinction through the vegetation layer and 2) surface roughness which decreases the reflectivity of the ground surface.

From these analyses, it is found that the backscatter at $\mathrm{C}$-band or higher frequencies is mainly sensitive to vegetation parameters for sufficiently high vegetation biomass (in this case, biomass $=1.97 \mathrm{~kg} / \mathrm{m}^{2}$ ). At L-band or lower frequencies, it is possible to sense the soil moisture for surfaces covered with short vegetation and relatively high biomass. Fig. 12(a)-(c) 


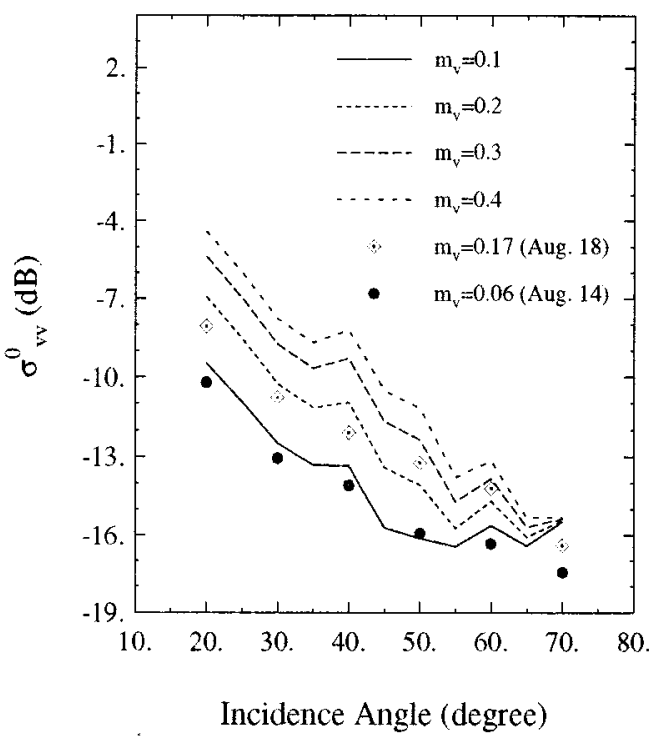

(a)

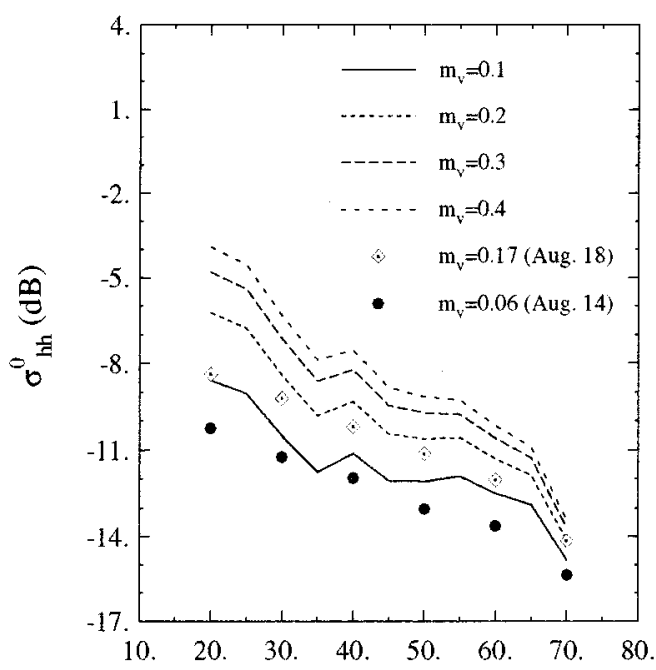

Incidence Angle (degree)

(b)

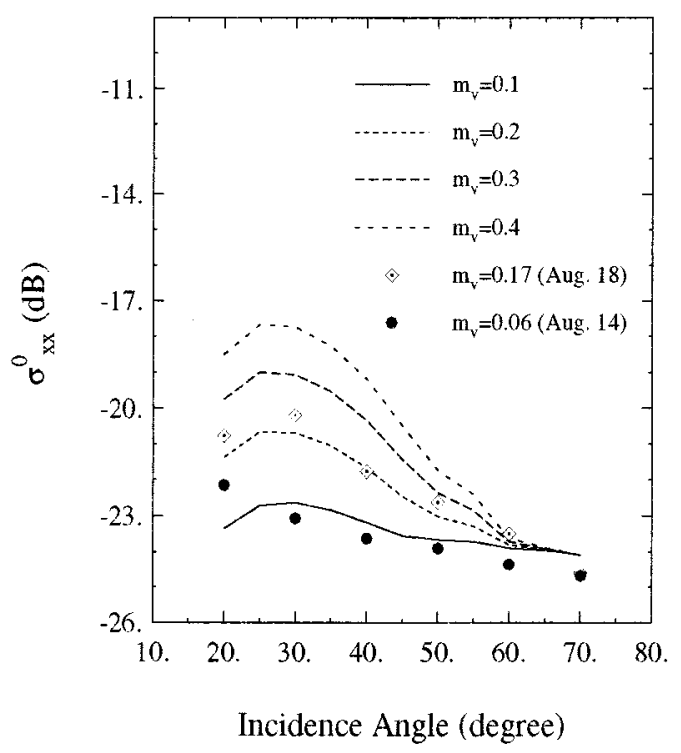

(c)

Fig. 12. Analysis of sensitivity to the variation of the soil moisture for the POLARSCAT data set at L-band: (a) vv-polarization, (b) hh-polarization, and (c) cross-polarization.

demonstrate the sensitivity of the backscatter to soil moisture as a function of incidence angle for the soybean field. The simulations are performed under four different soil-moisture conditions: $m_{v}=0.1,0.2,0.3$, and 0.4 at L-band. The backscatter data collected on August 14 and 18 are also plotted in these figures for comparison. These results suggest that the appropriate range of incidence angle for the purpose of soil-moisture retrieval is $\theta_{i}<50^{\circ}$, where there is about $6 \mathrm{~dB}$ of dynamic range. At incidence angles larger than $50^{\circ}$, the sensitivity to soil moisture decreases due to the high extinction caused by the vegetation. To retrieve the soil moisture accurately, vegetation parameters must be estimated as accurately as possible. It seems a combination of high and low frequency backscatter data is needed to estimate the vegetation and soil moisture accurately.

Due to the limited ground truth data, the AIRSAR data set is used for estimating the vegetation and surface roughness parameters. Although the retrieval algorithm presented here is based on trial and error, it indicates the feasibility of estimating vegetation parameters and soil moisture from image radars. The procedure for estimating these parameters is described as follows.

1) Based on a series of trial simulations, it is found that the second-order near-field interaction can be ignored at L-band and C-band for the one-month old soybeans. In this case, the soybean plants are still young, with shorter branches and stems and much fewer numbers of vegeta- 


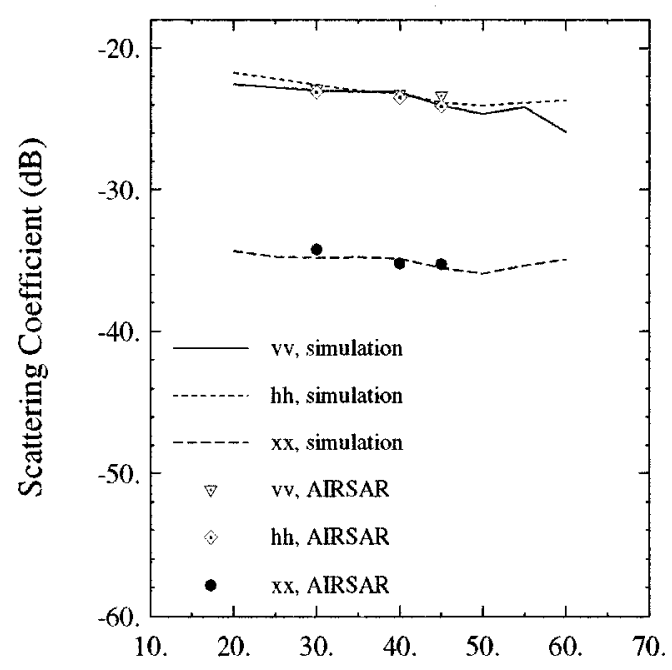

Incidence Angle (degree)

(a)

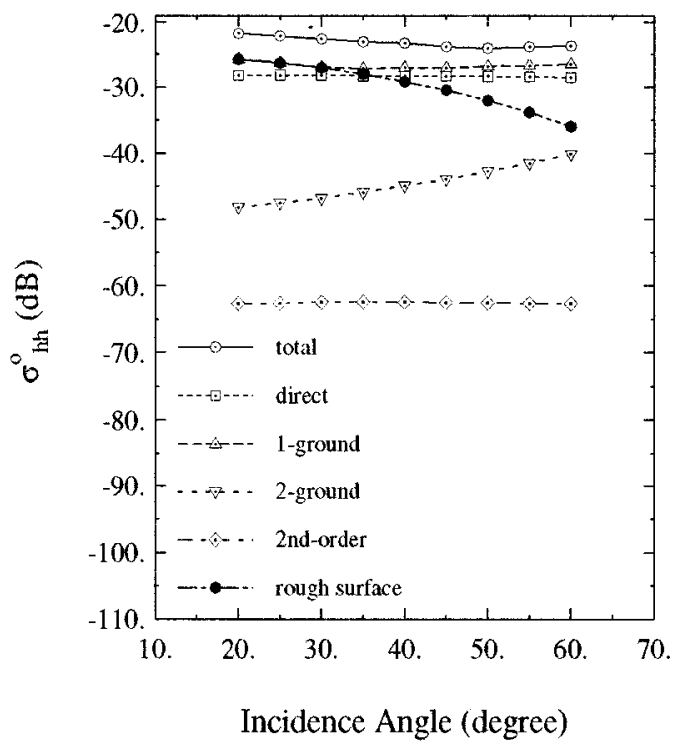

(c)

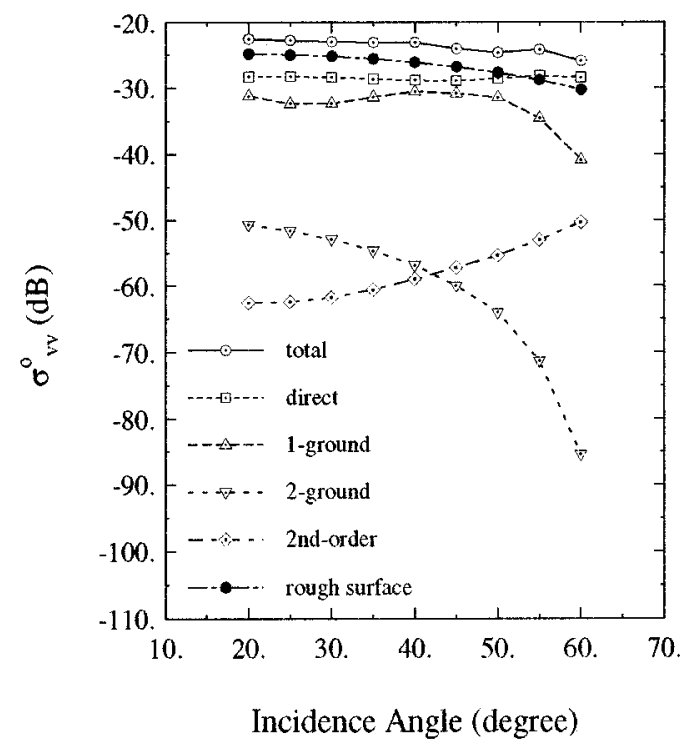

(b)

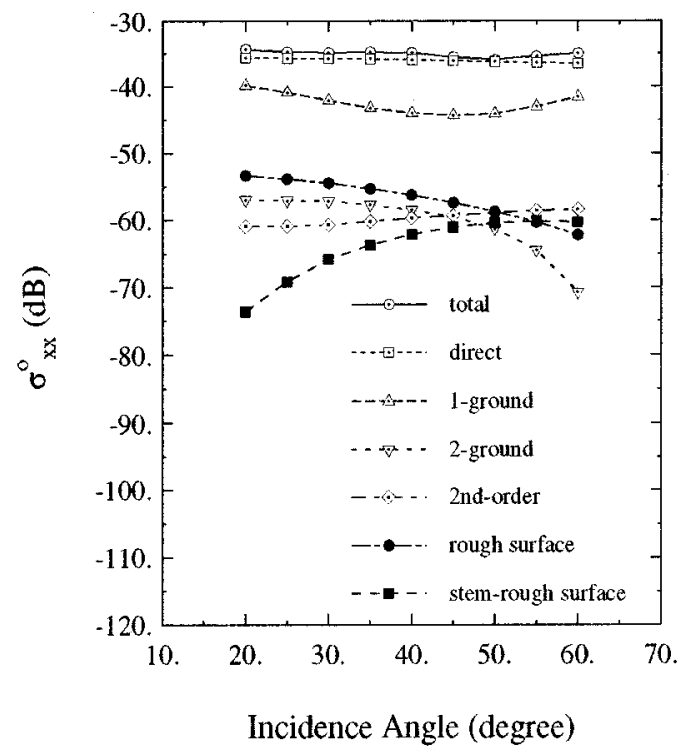

(d)

Fig. 13. Scattering coefficients versus incidence angle at L-band for AIRSAR dataset: (a) model validation and scattering mechanism analysis for (b) vv-polarizations, (c) hh-polarizations, and (d) cross-polarizations.

tion particles. Also, there are no pods on the plants, whose interaction with the main stem is the major source of the near-field interaction.

2) Judging from the measured values of the copolarized scattering coefficients reported in Fig. 13(a), it is inferred that the vegetation biomass is rather low. In this case, depending on the surface roughness, the surface scattering mechanism can be dominant at low incidence angles. If the surface scattering is dominant entirely, it is expected that $\sigma_{v v}^{\circ}$ will be larger than $\sigma_{h h}^{\circ}$. However, this is not observed from the measured data at $30^{\circ}$. Hence, there is at least a comparable backscattering contribution from the vegetation. Under this condition, a significant contribu- tion to the backscatter at C-band comes from the vegetation.

3) At relatively low biomass, it is found that the cross-polarized scattering coefficient is dominated by the direct backscatter from the soybean at both frequency bands. The size of the main stems for one-month-old soybean is small, so the rough surface-stem interaction is not significant. Also, at C-band, the direct backscatter from the rough surface is weak due to the small root mean square (RMS) height and extinction through the vegetation layer. Therefore, the dimension, the number density, and the dielectric constant of the soybean can be estimated by matching the cross-polarized backscatter at $\mathrm{C}$-band. This 


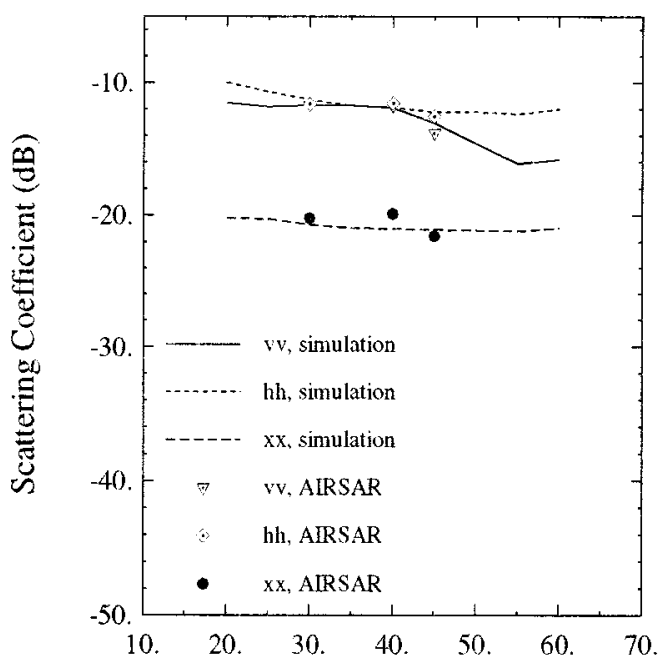

Incidence Angle (degree)

(a)

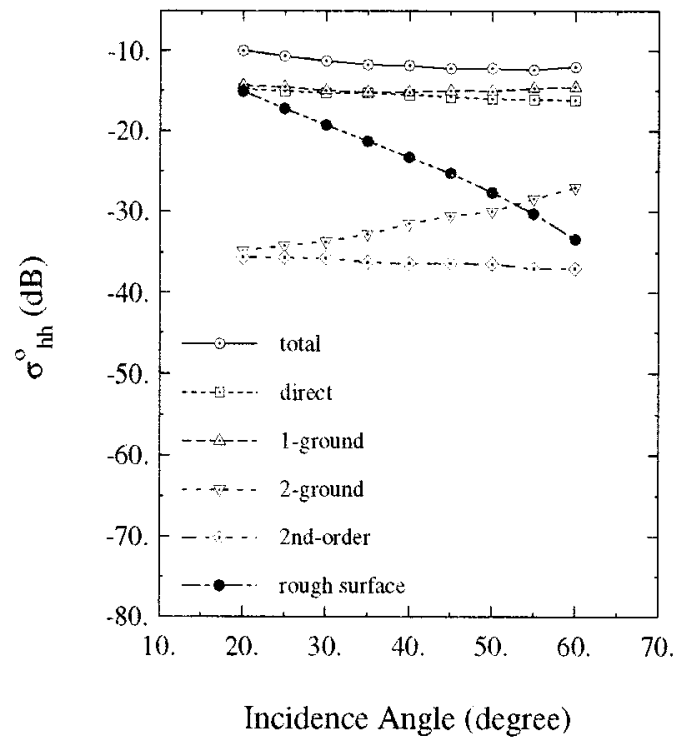

(c)

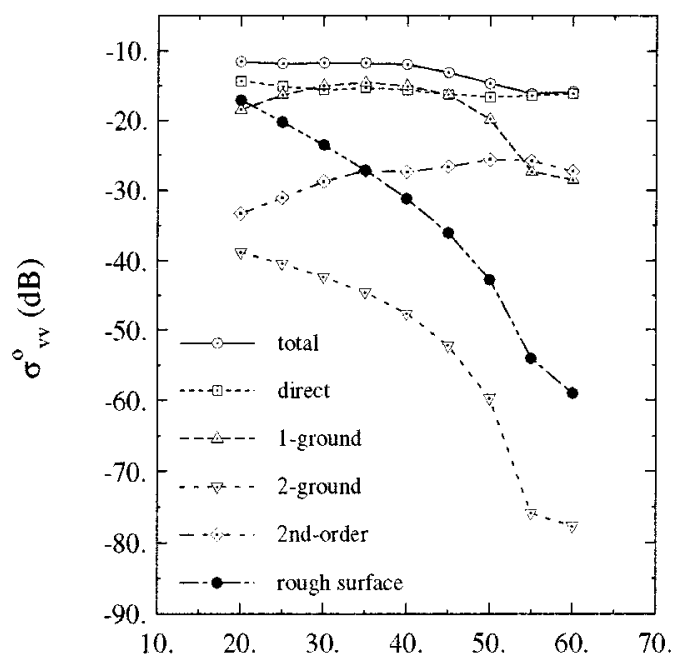

Incidence Angle (degree)

(b)

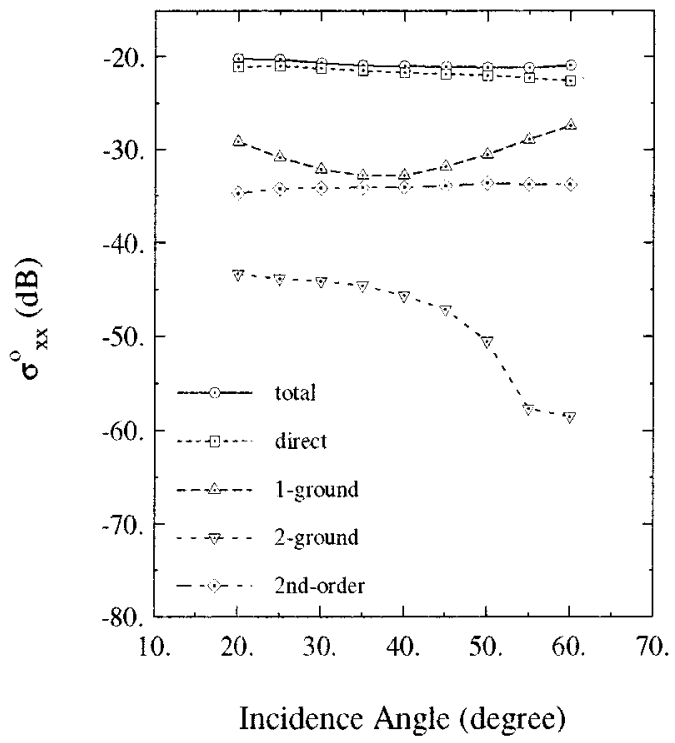

(d)

Fig. 14. Scattering coefficients versus incidence angle at C-band for AIRSAR dataset: (a) model validation and scattering mechanism analysis for (b) vv-polarizations, (c) hh-polarizations, and (d) cross-polarizations.

TABLE III

ESTIMATED GROUND TRUTH FOR THE AIRSAR DATA SET

\begin{tabular}{c|c}
\hline soil $\left(m_{v}\right)$ & 0.05 \\
\hline rms height $(s)$ & $0.0038 \mathrm{~m}$ \\
\hline correlation length $(l)$ & $0.038 \mathrm{~m}$ \\
\hline number density of plant & 19 plants $/ \mathrm{m}^{2}$ \\
\hline biomass & $0.22 \mathrm{~kg} / \mathrm{m}^{2}$ \\
\hline
\end{tabular}

is done by confining the range of the vegetation dielectric constants to those reported in Fig. 6. The elevation angles of all vegetation particles can be estimated by matching the copolarized scattering coefficient ratio $\sigma_{v v}^{0} / \sigma_{h h}^{0}$ and cross-polarized scattering coefficient. The vegetation parameters as a first iteration are decided by matching the data at C-band. Then, by matching the data at L-band with the same vegetation structure, the parameters of the rough surface are estimated. The simulation is then iterated between L-band and C-band, until the simulated and measured data match at both frequency bands.

After matching the backscatter data at both L-band and C-bands, the final estimated target parameters are shown in Tables III and IV. A typical corresponding computer-generated soybean plant is shown in Fig. 9(b). Figs. 13(a) and 14(a) show the simulated and measured scattering coefficients versus incidence angle at L-band and C-band, respectively. A Monte Carlo simulation is performed at $5^{\circ}$ increments. Fig. 13(b)-(d) shows scattering contributions from different mechanisms versus incidence angle at L-band. As predicted, the scattering 


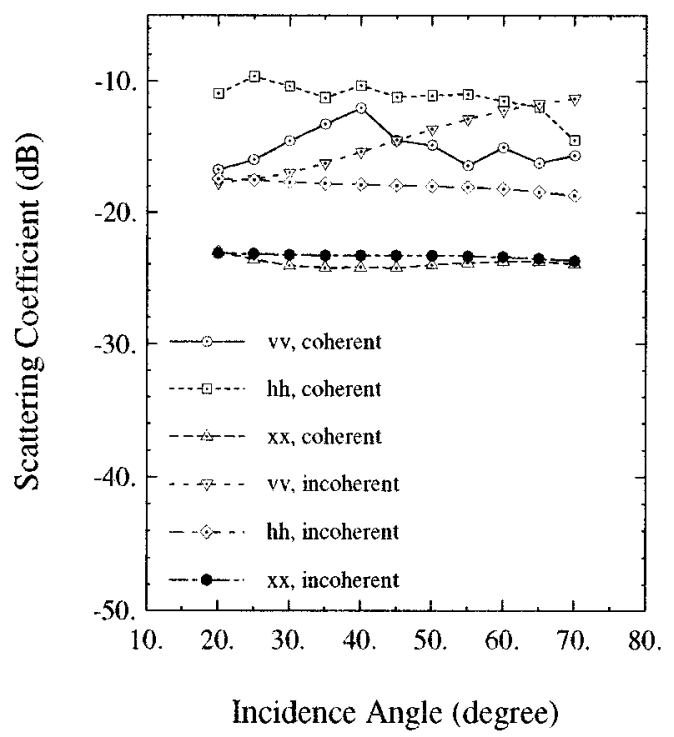

(a)

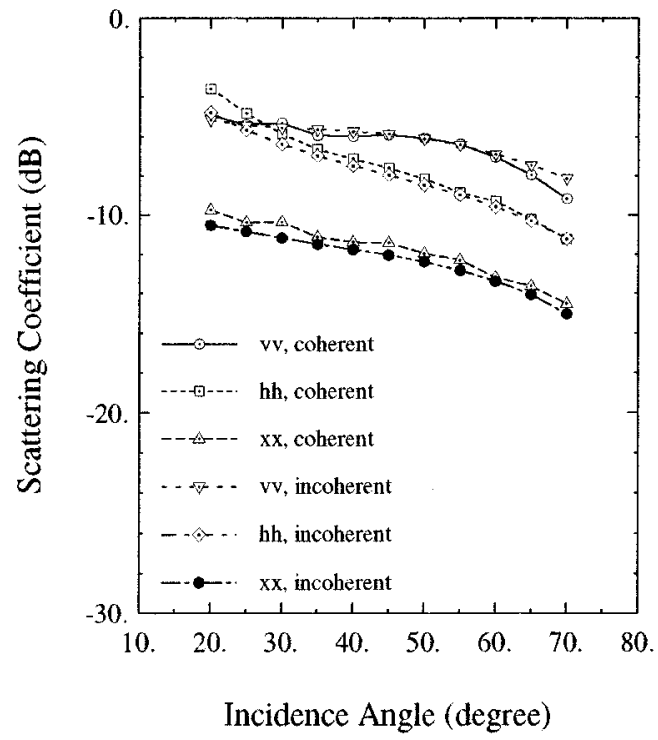

(b)

Fig. 15. Demonstration of the coherence effect caused by the soybean plant structure for a fully grown soybean field at (a) L-band and (b) C-band.

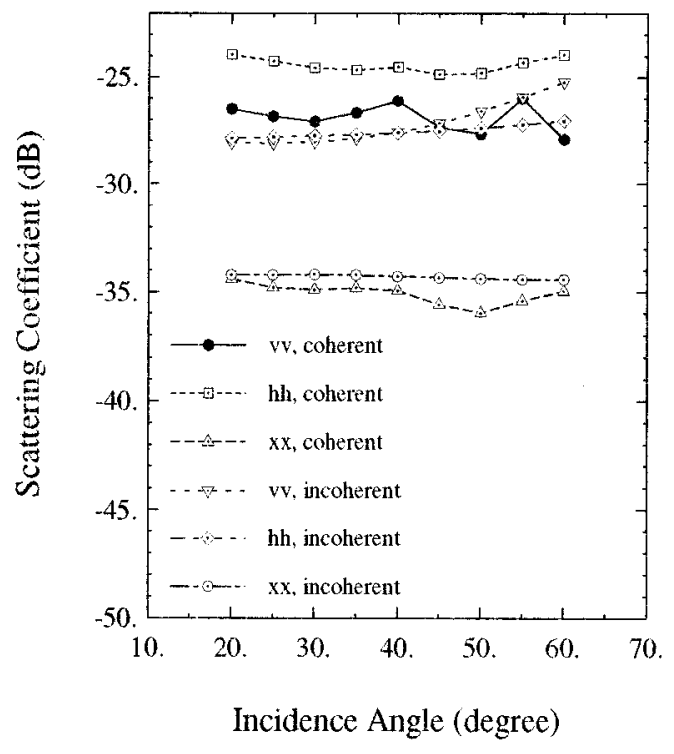

(a)

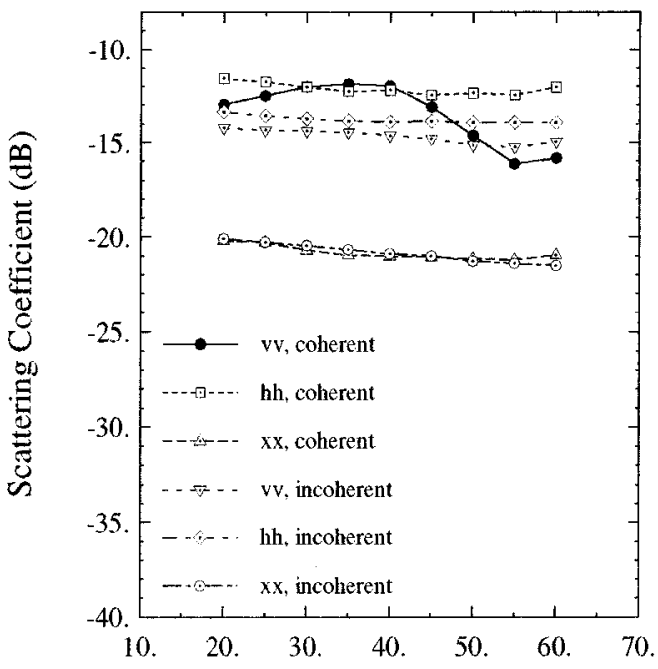

Incidence Angle (degree)

(b)

Fig. 16. Demonstration of the coherence effect caused by the soybean plant structure for a young soybean field at (a) L-band and (b) C-band.

TABLE IV

ESTIMATED Vegetation PARAMETERS OF SOYBEANS FOR THE AIRSAR DATA SET

\begin{tabular}{c|c|c|c}
\hline & $\beta_{m}, \beta_{s}$ (degree) & radius $(\mathrm{cm})$ & length or thickness $(\mathrm{cm})$ \\
\hline stem & $7.5,5$ & $0.18 \pm 0.05$ & $30.2 \pm 3.4$ \\
\hline node & $7.5,5$ & $0.18 \pm 0.05$ & $5.0 \pm 1.0$ \\
\hline branch & $60.8,25.6$ & $0.12 \pm 0.031$ & $14.7 \pm 4.5$ \\
\hline leaf & $47.0,30.0$ & $3.7 \pm 0.08(0.6)$ & $0.02 \pm 0.001$ \\
\hline
\end{tabular}

between stems and rough surface is not significant due to the shorter and slimmer main stems and smaller surface roughness. Fig. 13(b)-(d) shows scattering contributions from different mechanisms versus incidence angle at C-band. As predicted, the second-order scattering can be neglected.

Finally, Figs. 15 and 16 show the coherence effect of the vegetation structure. The scattering coefficients do not include the contribution from the main stems' rough surface scattering and the direct backscatter from the rough surface. In these figures, the coefficients denoted as "coherent" are calculated using (26), while those denoted as "incoherent" are calculated using (29). It is shown that for a fully grown soybean, the coherence effect is significant at L-band for copolarized components, while the effect is not observable at C-band. However, for low biomass condition (AIRSAR data), it is found that the coherent effect is also significant at $\mathrm{C}$-band. This can be explained by noting 
that a fully-grown soybean plant has more complex structure with more particles than a one-month-old plant. Nevertheless, it should be noted that the second-order near-field interaction is significant for POLARSCAT data at C-band and can be evaluated only when the relative distance and orientation of particles are given. Therefore, to some extent, the coherence effect of structure embedded in this mechanism is also important at $\mathrm{C}$-band. For the cross-polarized scattering, the coherence effect is less significant in both low and high biomass conditions at both frequencies.

\section{CONCLUSIONS}

In this paper, an electromagnetic scattering model for short branching vegetation is presented. The vegetation particles are modeled as simple geometries such as cylinders and disks, for which analytical scattering solutions are available. With realistic structures that reasonably describe the relative positions of the particles, this model is constructed so that the coherence effect due to the phase difference between the scattered fields from different particles and the second-order near-field interaction among particles are accounted for. Also, the interaction between the main stems and underlying rough surface is incorporated into this model, which is shown to be important only at low frequencies (L-band) and for cross-polarized backscattering coefficient.

The model accuracy is verified using polarimetric radar backscatter measurements of a soybean field obtained from truck-mounted scatterometers. Through an extensive ground truth data collection, target parameters such as the soil and vegetation moisture contents, geometry of the soybean plants, and surface roughness were characterized. Monte Carlo simulations were carried out simulating the statistical properties of the backscatter at different incidence angles. Good agreement is obtained between the model prediction and measured backscattering coefficients. From a sensitivity analysis, the following is found.

1) Second-order near-field interaction is more significant at C-band than at L-band.

2) The interaction between the main stems and rough surfaces could be significant for cross-polarized scattering at L-band.

3) The double ground-bounce mechanism is generally not important.

4) High-frequency data (C-band or higher) can be used to probe the vegetation, and low-frequency data (L-band or lower) is needed to probe the soil moisture through vegetation.

This model was also used to estimate the parameters of a soybean field using the AIRSAR data, and reasonable results that agree with the limited ground truth data have been obtained. The coherence effect was also examined using the model simulation.

\section{REFERENCES}

[1] F. T. Ulaby, K. Sarabandi, K. McDonald, M. Whitt, and M. C. Dobson, "Michigan microwave canopy scattering model," Int. J. Remote Sensing, vol. 11 , no. 7 , pp. $2097-2128,1990$
[2] M. A. Karam and A. K. Fung, "Electromagnetic scattering from a layer of finite length, randomly oriented, dielectric circular cylinders over a rough interface with application to vegetation," Int. J. Remote Sensing, vol. 9, pp. 1109-1134, 1988 .

[3] R. H. Lang and J. S. Sidhu, "Electromagnetic backscattering from a layer of vegetation: A discrete approach," IEEE Trans. Geosci. Remote Sensing, vol. 21, pp. 62-71, 1983.

[4] G. Zhang, L. Tsang, and Z. Chen, "Collective scattering effects of trees generated by stochastic Lindenmayer systems," Microwave Opt. Technol. Lett., vol. 11, pp. 107-111, Feb. 1995.

[5] S. H. Yueh, J. A. Kong, J. K. Jao, R. T. Shin, and T. L. Toan, "Branching model for vegetation," IEEE Trans. Geosci. Remote Sensing, vol. 30, pp. 390-402, Mar. 1992.

[6] Y. C. Lin and K. Sarabandi, "A Monte Carlo coherent scattering model for forest canopies using fractal generated trees", submitted for publication.

[7] K. Sarabandi and P. F. Polatin, "Electromagnetic scattering from two adjacent objects," IEEE Trans. Antennas Propagat., vol. 42, pp. 510-517, Apr. 1994.

[8] L. Tsang, K. Ding, G. Zhang, C. C. Hsu, and J. A. Kong, "Backscattering enhancement and clustering effects of randomly distributed dielectric cylinders overlying a dielectric half space based on Monte Carlo simulations," IEEE Trans. Antennas Propagat., vol. 43, pp. 488-499, May 1995.

[9] P. H. Raven, R. F. Evert, and S. E. Eichhorn, Biology of Plants. New York: Worth, 1986

[10] T. Chiu and K. "Sarabandi, Electromagnetic scattering interaction between a dielectric cylinder and a slightly rough surface", submitted for publication.

[11] A. Ishimaru, Wave Propagation and Scattering in Random Media. New York: Academic, 1978, vol. 2.

[12] R. Schiffer and K. O. Thielheim, "Light scattering by dielectric needles and disks," J. Appl. Phys., vol. 50, Apr. 1979.

[13] K. Sarabandi and T. B. A. Senior, "Low-frequency scattering from cylindrical structures at oblique incidence," IEEE Trans. Geosci. Remote Sensing, vol. 28, pp. 879-885, Sept. 1990.

[14] L. Tsang, J. Kong, and R. T. Shin, Theory of Microwave Remote Sensing. New York: Wiley, 1985.

[15] S. S. Seker and A. Schneider, "Electromagnetic scattering from a dielectric cylinder of finite length," IEEE Trans. Antennas Propagat., vol. 36, pp. 303-307, Feb. 1988.

[16] P. F. Polatin, K. Sarabandi, and F. T. Ulaby, "Monte Carlo simulation of electromagnetic scattering from a heterogeneous two-component medium," IEEE Trans. Antennas Propagat., vol. 43, pp. 1048-1057, Oct. 1995.

[17] K. Sarabandi and T. Chiu, "Electromagnetic scattering from slightly rough surface with inhomogeneous dielectric profiles," IEEE Trans. Antennas Propagat., vol. 45, pp. 1419-1430, Sept. 1997.

[18] F. T. Ulaby, R. K. More, and A. K. Fung, Microwave Remote Sensing: Active and Passive. Norwood, MA: Artech House, 1982, vol. 2.

[19] T. Chiu and K. "Sarabandi, Electromagnetic scattering interaction between a dielectric cylinder and a slightly rough surface", submitted for publication

[20] J. Stiles, "A coherent polarimetric microwave scattering models for grassland structures and canopies," Ph.D. dissertation, Univ. Michigan, Ann Arbor, 1996.

[21] M. A. Tassoudji, K. Sarabandi, and F. T. Ulaby, "Design consideration and implementation of the LCX polarimetric scatterometer (POLARSCAT)," Radiation Lab., Univ. Michigan, Ann Arbor, Rep. 022 486-T-2, June 1989.

[22] D. R. Brunfeldt, "Theory and design of a field-portable dielectric measurement system," IEEE Int. Geoscience and Remote Sensing Symp. (IGARSS) Dig., vol. 1, pp. 559-563, 1987.

[23] M. T. Hallikainen, F. T. Ulaby, M. C. Dobson, M. A. El-Rayes, and L. Wu, "Microwave dielectric behavior of wet soil—Part I: Empirical models and experimental observations," IEEE Trans. Geosci. Remote Sensing, vol. GE-23, pp. 25-34, Jan. 1985.

[24] K. Sarabandi and F. T. Ulaby, "Technique for measuring the dielectric constant of thin materials," IEEE Trans. Instrum. Meas., vol. 37, pp. 631-636, Dec. 1988.

[25] K. Sarabandi, "A technique for dielectric measurement of cylindrical objects in a rectangular waveguide," IEEE Trans. Instrum. Meas, vol. 43, pp. 793-798, Dec. 1994.

[26] F. T. Ulaby and M. A. El-Rayes, "Microwave dielectric spectrum of vegetation-Part II: Dual-dispersion model," IEEE Trans. Geosci. Remote Sensing, vol. GE-25, pp. 550-557, 1987.

[27] Online: http://www.jpl.nasa.gov/mip/airsar.html 


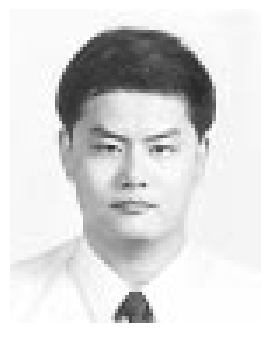

Tsenchieh Chiu received the B.S. and M.S. degrees from National Taiwan University, Taipai, Taiwan, R.O.C. in 1990, and 1992, respectively, and the $\mathrm{Ph} . \mathrm{D}$. degree from the University of Michigan, Ann Arbor, 1998

His research interests include microwave remote sensing, eletromagnetic scattering from rough surfaces and vegetation, and pattern synthesis of antenna array.

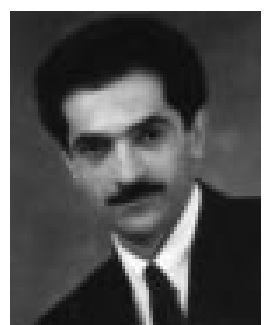

Kamal Sarabandi (S'87-M'90-SM'92-F'00) received the B.S. degree in electrical engineering from Sharif University of Technology, Tehran, Iran, in 1980, and the M.S.E. degree in electrical engineering in 1986 and the M.S. degree in mathematics and the $\mathrm{Ph} . \mathrm{D}$. degree in electrical engineering in 1989, all from the University of Michigan, Ann Arbor, MI.

From 1980 to 1984, he worked as a Microwave Engineer in the Telecommunication Research Center, Sharif University of Technology. He is currently an Associate Professor in the Department of Electrical Engineering and Computer Science with the University of Michigan. He has 18 years of experience with microwave sensors and radar systems. In the past eight years, he has served as the Principal Investigator and Coinvestigator on many projects sponsored by NASA, JPL, ARO, ONR, ARL, and GM, all related in one way or another to microwave and millimeter wave radar remote sensing. $\mathrm{He}$ has published many book chapters and more than 80 papers in refereed journals on electromagnetic scattering, random media modeling, microwave measurement techniques, radar calibration, application of neural networks in inverse scattering problems, and microwave sensors. He has also had more than 140 papers and invited presentations in national and international conferences and symposia on similar subjects.

Dr. Sarabandi is listed in American Men and Women of Science and Who's Who in Electromagnetics. He has been a Member of the IEEE Geoscience and Remote Sensing AdCom since January of 1998 and has served as the Chairman of the Geoscience and Remote Sensing Society's Southeastern Michigan chapter from 1992 to 1998. He is also a Member of Commission F of URSI and of the Electomagnetic Academy. He was a recipient of a 1996 Teaching Excellence Award, the 1997 Henry Russel Award from the Regent of the University of Michigan, and the 1999 GAAC Distinguished Lecturer Award from the German Federal Ministry for Education, Science, and Technology. 